\title{
La pandemia africana: passato, presente e futuro
}

\author{
Chiara Zavattero
}

UO di Pediatria, Ospedale G.B. Morgagni - L. Pierantoni, AUSL della Romagna, Forlì

\section{2020}

Il primo caso confermato di infezione da SARS-CoV-2 nel continente africano risale al 14 febbraio 2020, in Egitto. Il 24 maggio 2020, a circa due mesi dalla promozione dell'infezione da SARS-CoV-2 a pandemia, le curve epidemiche di America, Asia ed Europa crescevano vertiginosamente mentre quella africana si manteneva piatta, nonostante il CFR (Case Fatality Ratio), ossia il rapporto tra il numero dei decessi e il numero dei casi confermati, fosse assai simile a quello asiatico (Tabella 1) [1]. Al 6 luglio 2021 i casi di Covid nel continente africano hanno superato i 4 milioni, con un totale di circa 100.000 morti, numeri che seppur in crescita si mantengono molto al di sotto degli standard internazionali. Il CFR africano sembra non avere mai subito sostanziali variazioni, mantenendosi stabilmente attorno al 3\% (Figura 1) [2].

Questa anomalia ha destato l'interesse di medici, biologi e statisti. In un continente caratterizzato da una popolazione fragile (elevata incidenza di HIV, TBC e malaria, malnutrizione diffusa e condizioni di estrema povertà), da un sistema economico e sanitario ancora deboli, da scarse capacità diagnostiche per carenza di risorse umane e tecnologiche, dall'esistenza di aree urbane densamente popolate e con scadenti condizioni igieniche nonché da modelli politici eterogenei che hanno predisposto misure profilattiche difformi, ci si sarebbe aspettato un andamento epidemico ben diverso.

L'American Society of Tropical Medicine and Hygiene si è interrogata sulle possibili cause di questa anomalia, identificando quattro ipotesi principali [3]:

- le restrizioni imposte ai viaggi internazionali;

- la bassissima età media della popolazione (<20 anni);

- il clima secco e caldo (media diurna sub-sahariana $>20{ }^{\circ} \mathrm{C}$ durante tutto l'anno; ridotta stabilità del virus e riduzione del titolo virale a $23-25^{\circ} \mathrm{C}$ );

- la possibile preesistente immunizzazione delle popolazioni africane (contatti con animali vettori, cross-reattività anticorpale da analogia antigenica con bat-SARS-like coronavirus, 85\%, e SARS-CoV-1, 76\%).

Se da un lato una popolazione giovane (per il 75\% < 35 anni) vede di molto ridotta l'incidenza delle forme più gravi di infezione, dall'altro queste potrebbero essere favorite dall'elevata prevalenza di comorbilità legate a HIV, TBC e malaria. Tuttavia, mentre i soggetti giovani a potenziale rischio di infezione asintomatica possono diventare vettori di diffusione del virus, i soggetti affetti da HIV, TBC e malaria contraggono infezioni a decorso rapido e spesso sfavorevole, con ridotta probabilità di trasmettere a loro volta il virus [4]. Anche la distribuzione rurale della popolazione ha certamente contribuito ad abbattere i picchi epidemici attesi: un'elevata percentuale di africani abita in aree periferiche in cui lo stile di vita si associa a un naturale distanziamento sociale con ridotta mescolanza della popolazione [5].
Non manca chi sostiene che la sottostima dei dati provenienti dal continente africano sia legata all'insufficiente screening della popolazione, sintomatica e non. Gli epidemiologi lamentano la povertà di dati provenienti da Africa settentrionale e Africa centrorientale e la loro bassa qualità legata alla mancata stratificazione per caratteristiche demografiche e di comorbilità [6]. L'irrequietezza politica di queste aree e la presenza di un gran numero di rifugiati e profughi complica la situazione. Senza l'intervento dell'OMS e di altre organizzazioni internazionali, la raccolta dati sul Covid-19 sarebbe non solo molto più incompleta ma anche di scarso utilizzo ai fini dell'identificazione dei fattori di rischio e dei punti deboli sui quali programmare campagne di intervento sociale e sanitario.

\section{Misure di contenimento}

Da aprile 2020 anche i Paesi africani hanno introdotto misure di contenimento ge-

\begin{tabular}{|l|l|l|}
\hline \multicolumn{2}{|c|}{ TABELLA 1. Numero di contagi e CFR (Case Fatality Ratio) nei continenti } \\
americano, asiatico, europeo e africano, aggiornati al 24 maggio 2020 \\
\hline & SOGGETTI CONTAGIATI & CFR \\
\hline Americhe & 2.420 .000 & $5,9 \%$ \\
\hline Asia & 927.000 & $2,9 \%$ \\
\hline Europa & 1.810 .000 & $9,3 \%$ \\
\hline Africa & 108.000 & $3 \%$ \\
\hline
\end{tabular}

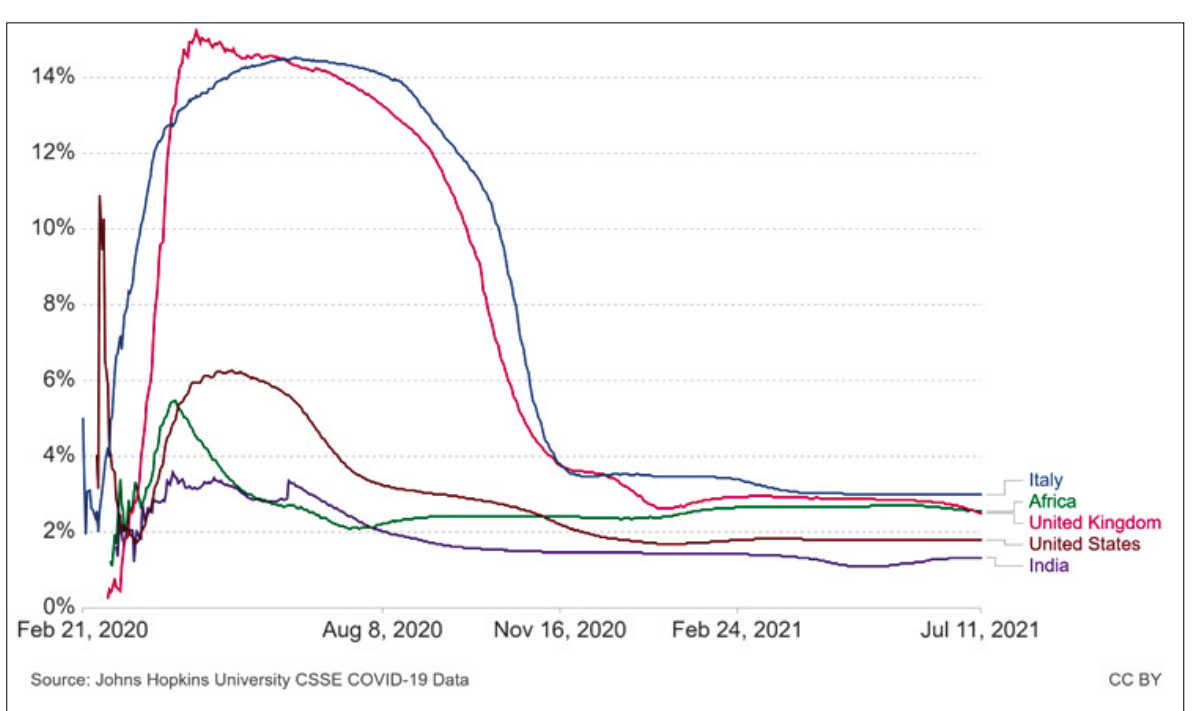

Figura 1. CFR di Italia, Africa, UK, USA e India nell'intervallo febbraio 2020-luglio 2021 (https://ourworIdindata.org). 
ografico (blocco dei voli internazionali), di quarantena a domicilio e di restrizione degli incontri pubblici (scuole, chiese, ristoranti, eventi sportivi), ma a giugno 2020 in alcuni Paesi si assisteva già all'alleggerimento delle misure profilattiche. È accaduto così che nello stesso continente l'epidemia si sia sviluppata con dinamiche estremamente diversificate.

In Nordafrica, Tunisia e Marocco hanno rapidamente arginato il contagio avviando precoci misure di isolamento, con riapertura dei confini a luglio 2020. In Egitto, primo Paese africano toccato dal virus, si è invece attesa la conclusione della festività di Eid (fine maggio) per imporre misure contenitive, tanto che attualmente il Paese occupa la seconda posizione nella classifica africana di incidenza e mortalità [7-8].

Nell'Africa subsahariana, Uganda e Sudafrica hanno imposto misure più restrittive, ciononostante il Sudafrica risulta ancora la nazione più colpita del continente. È possibile che la maggiore capacità di screening abbia favorito, più che altrove, l'emergere del fenomeno. Nei fatti, a giugno 2020, mentre il Sudafrica allestiva centri di diagnosi e si attrezzava per affrontare l'emergenza, in Tanzania il presidente Magufuli dichiarava il Paese Covid-free "due to the grace of God". Attualmente il Sudafrica batte in incidenza la Tanzania 4.000:1 ma se guardiamo al CFR dei due Paesi (Sudafrica 3\% vs Tanzania 4,1\%) nasce il sospetto di un'epidemia tanzaniana attiva ma sotto diagnosticata (Figura 2). Fortunatamente l'approccio della nuova presidentessa $\mathrm{Sa}-$ mia Suluhu sembra essere più scientifico di quello del suo predecessore, deceduto a marzo 2021 in circostanze poco chiare.

Posta l'eterogenea e talvolta discordante scelta di misure profilattiche e contenitive nei diversi Paesi africani, la loro concreta applicazione ha risentito di alcuni fattori [5]:

- la scarsità delle risorse;

- l'eterogenea rappresentazione culturale e religiosa della popolazione africana, mondo sciamanico incluso;

- la scarsa regolarizzazione del mondo del lavoro e la quasi totale assenza di servizi sociali di supporto alla popolazione che hanno reso impossibile per molti la sospensione dell'attività lavorativa;

- le precarie condizioni igieniche: la maggior parte della popolazione si serve di bagni comuni rendendo di fatto irrealizzabile l'isolamento domiciliare.

\section{2021}

Nelle ultime settimane la terza ondata sta interessando tutto il continente comportando un rapido incremento dei casi di malattia grave e delle morti correlate. Il numero di casi raddoppia ogni 3 setti- mane, superando il picco di positività del luglio 2020 (Figura 3) [9-10].

Cosa è cambiato?

È probabile che molti Paesi abbiano intensificato lo screening della popolazione.

Le misure di contenimento non si riescono a integrare con la quotidianità della popolazione africana, specialmente nelle aree urbane più povere e affollate.

Nell'emisfero sud stanno attualmente vivendo la stagione invernale che, sebbene più mite, potrebbe predisporre a una maggiore diffusione del virus.

Le nuove e più contagiose varianti virali sono arrivate anche qui. La variante Delta, in particolare, è stata identificata in 16 nazioni africane ed è attualmente dominante in Sudafrica.

\section{Effetti collaterali della pandemia}

Come osservato in altre aree del mondo, la polarizzazione dei servizi sanitari sulla diagnosi e trattamento dell'epidemia di
Covid-19, assieme al timore della popolazione ad avvicinarsi alle strutture ospedaliere, ha finito per interferire pesantemente con il mantenimento dei servizi di salute essenziali. Il rallentamento delle campagne internazionali di supporto e sviluppo relative alla salute sessuale e al settore materno-infantile rischia di avere un enorme impatto negativo non solo sulla salute della popolazione africana ma anche sullo sviluppo economico e socioculturale del continente.

Si temono concretamente una diminuzione della copertura vaccinale infantile con ricomparsa di focolai epidemici prevenibili, l'interruzione dei programmi di pianificazione familiare e di assistenza alla gravidanza con aumento di gravidanze indesiderate o complicate, un calo dell'assistenza specializzata al parto con l'aumento dei parti a domicilio, l'incremento di episodi di maltrattamento e di violenza domestica e l'aumento dei rapporti non protetti e dei casi di malattie sessualmente trasmissibili.

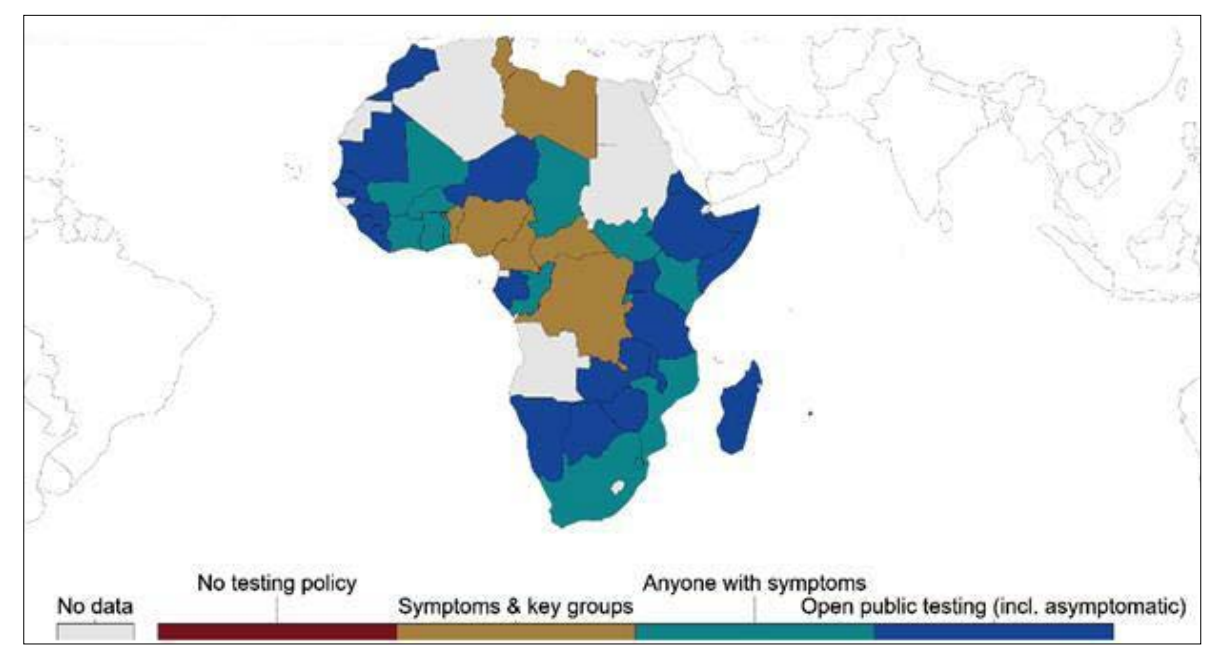

Figura 2. Politiche di screening per SARS-CoV-2 in Africa al 6 luglio 2021 (https://ourworldindata.org).

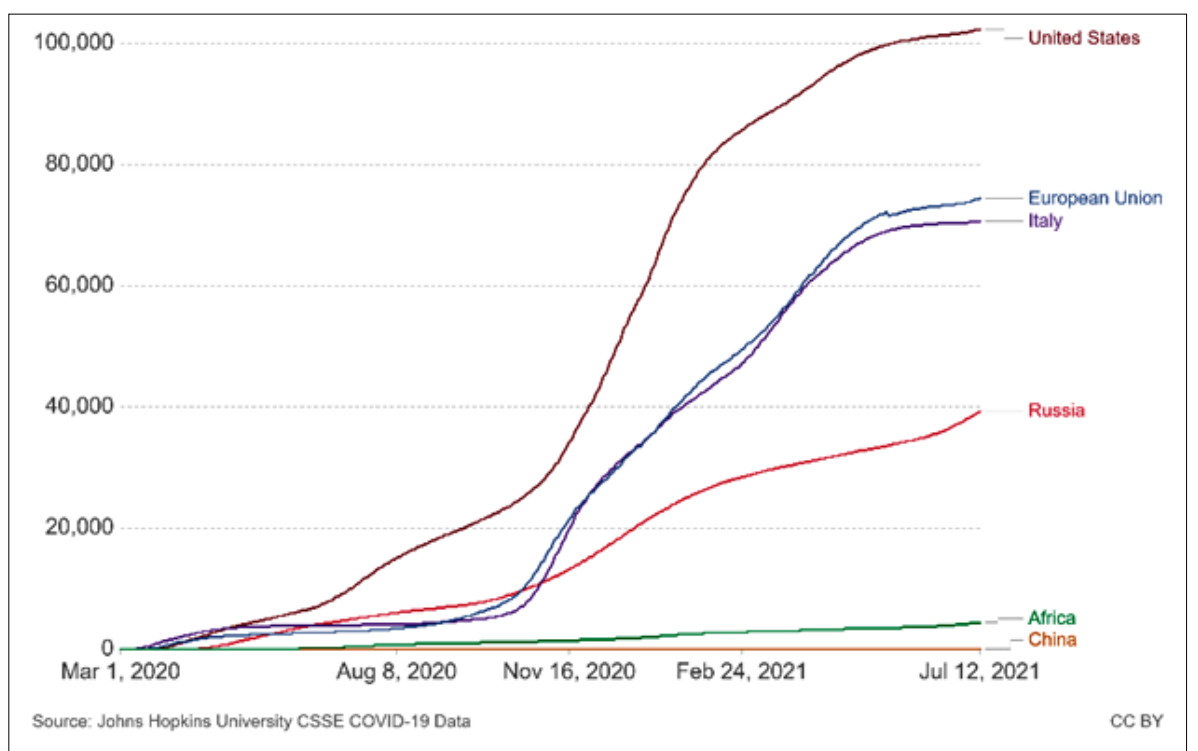

Figura 3. Casi cumulativi confermati di infezione da SARS-CoV-2 da marzo 2020 a luglio 2021 in Africa, Cina, Europa, Italia, Russia, USA (https://ourworldindata.org). 
Nei prossimi 5 anni si stima un incremento del 10-36\% della mortalità HIV-correlata che avrà come principali imputati l'interruzione delle terapie antiretrovirali, il ritardo diagnostico e il rallentamento delle campagne di educazione sanitaria [5]. Questa battuta d'arresto potrebbe non dipendere unicamente dall'atteggiamento della popolazione e dalle misure di lockdown imposte, ma anche dalla ridotta disponibilità di materiale e farmaci sia in termini di approvvigionamento nazionale che di capacità della popolazione di sostenere le spese mediche indispensabili.

Shapira e coll. [11] hanno studiato otto indicatori di utilizzo e funzionamento dei servizi a sostegno di riproduzione e salute materno-infantile (servizi ambulatoriali, vaccinazione pentavalente, vaccinazione con BCG, parto, prima e quarta visita in gravidanza, prima visita postnatale, pianificazione familiare) documentando gli accessi da gennaio 2018 a luglio 2020 in otto nazioni dell'Africa subsahariana.

Dall'arrivo del virus in Africa hanno dimostrato in ciascuno Stato un calo di attività per almeno uno degli otto servizi valutati, specialmente per quanto riguarda la vaccinazione dei bambini, l'accesso ospedaliero per prime visite e il numero di parti espletati in ambiente protetto.

Il calo delle vaccinazioni non è un dato da sottovalutare: in seguito all'epidemia di Ebola del 2013-2016 erano ricomparsi focolai epidemici di morbillo, di cui si teme una replica. Nello stesso periodo era incrementato del 30\% il numero dei parti espletati a domicilio e la sospensione degli incontri di educazione sanitaria della popolazione era stato seguito da un'incrementata incidenza della malaria.

L'I-SHARE team (International Sexual Health And REproductive health team) [12] ha avviato un progetto simile basato sulla somministrazione di questionari alla popolazione africana a tema sicurezza dei rapporti sessuali, violenza domestica, accesso ai servizi di salute riproduttiva, HIV testing, utilizzo di pratiche culturali eterolesive (es. mutilazioni genitali) e salute mentale. L'obiettivo è raccogliere dati affidabili che facilitino il confronto fra le condizioni di differenti nazioni e guidino gli interventi sanitari nei prossimi anni. $\mathrm{Ne}$ attenderemo il risultato.

\section{Parliamo di vaccinazioni}

Come prevedibile, la campagna vaccinale anti-Covid ha preso il via con una certa dose di ineguaglianza. I Paesi ricchi (Cina, USA, UK, Israele), grazie a specifici contratti stipulati con le aziende farmaceutiche, si sono garantiti precocemente la maggior parte delle dosi vaccinali prodot- te, mentre in altre aree del mondo (Africa, Sudamerica, Sudest asiatico) l'avvio delle vaccinazioni restava un miraggio.

Al $1^{\circ}$ luglio 2021, 51 Stati africani su 54 hanno avviato un piano vaccinale.

Sedici milioni di africani sono attualmente vaccinati: sembra un gran numero, eppure corrisponde a poco più dell' $1 \%$ della popolazione africana. In Italia è il 38,6\% della popolazione ad aver ricevuto entrambe le dosi (Figura 4).

C'è chi continua a sostenere che nonostante elevati livelli di produzione, un quarto della popolazione mondiale non avrà accesso al vaccino prima del 2022 [13].

Ma come arrivano i vaccini in Africa?

Non esistendo un vaccino di produzione locale, il continente dipende dai produttori stranieri. L'African Union si è assicurata 270 milioni di dosi, una briciola a fronte di una popolazione di 1,2 bilioni di persone. L'Africa dipende quindi anche in questo caso dalle donazioni, le quali si espletano secondo tre principali meccanismi [14]:

- "carità bilaterale": si tratta di donazioni da parte di nazioni che avanzano dosi vaccinali. È il sistema più debole in quanto non garantisce certezza né sulle tempistiche di fornitura né sulle dosi messe a disposizione;

- "carità multilaterale": rappresentata dal consorzio COVAX che riunisce WHO, CEO (Coalition for Epidemic Preparedness), United Nations Children's Fund e Gavi il cui scopo è accelerare l'accesso alla vaccinazione per i Paesi a medio e basso reddito. È stato stabilito che i Paesi ad alto reddito, a seconda del budget economico a disposizione, pos- sano richiedere dosi per il 10-50\% della loro popolazione e che per le stesse cifre i Paesi a basso e medio reddito ne ricevano per il 20\% della popolazione. Il consorzio COVAX si pone l'obiet tivo di coprire le spese per l'approvvigionamento di queste dosi. Pregevole senza dubbio, ma anche questo sistema mantiene il continente africano in uno stato di dipendenza dagli interventi altrui senza agire sui problemi sostanziali che sottendono questa necessità. $\mathrm{Nel}$ caso del vaccino anti-covid esiste inoltre il rischio che le dosi COVAX scivolino al fondo della lista di priorità, rallentando le campagne di vaccinazione, in attesa che tutti gli altri Paesi abbiano ricevuto quanto pagato;

- sospensione o rinuncia temporanea dell'Intellectual Property: si tratta di un'interruzione temporanea dei privilegi che le aziende farmaceutiche vantano sui brevetti vaccinali, così da permettere anche ad altre aziende di produrli. La Thailandia ha potuto sfruttare questo meccanismo, che purtroppo nei Paesi africani non sarebbe realizzabile sia per la mancanza di strutture adatte alla produzione sia perché per invogliare le aziende farmaceutiche alla condivisione delle informazioni servono incentivi finanziari, non disponibili.

La garanzia di un'equa distribuzione delle dosi vaccinali a tutte le nazioni è di fondamentale importanza ed è stata discussa in recenti incontri di dignitari internazionali (G7-G20) [15-16]: solo garantendo la copertura minima della popolazione mondiale arriveremo a rallentare la diffusione

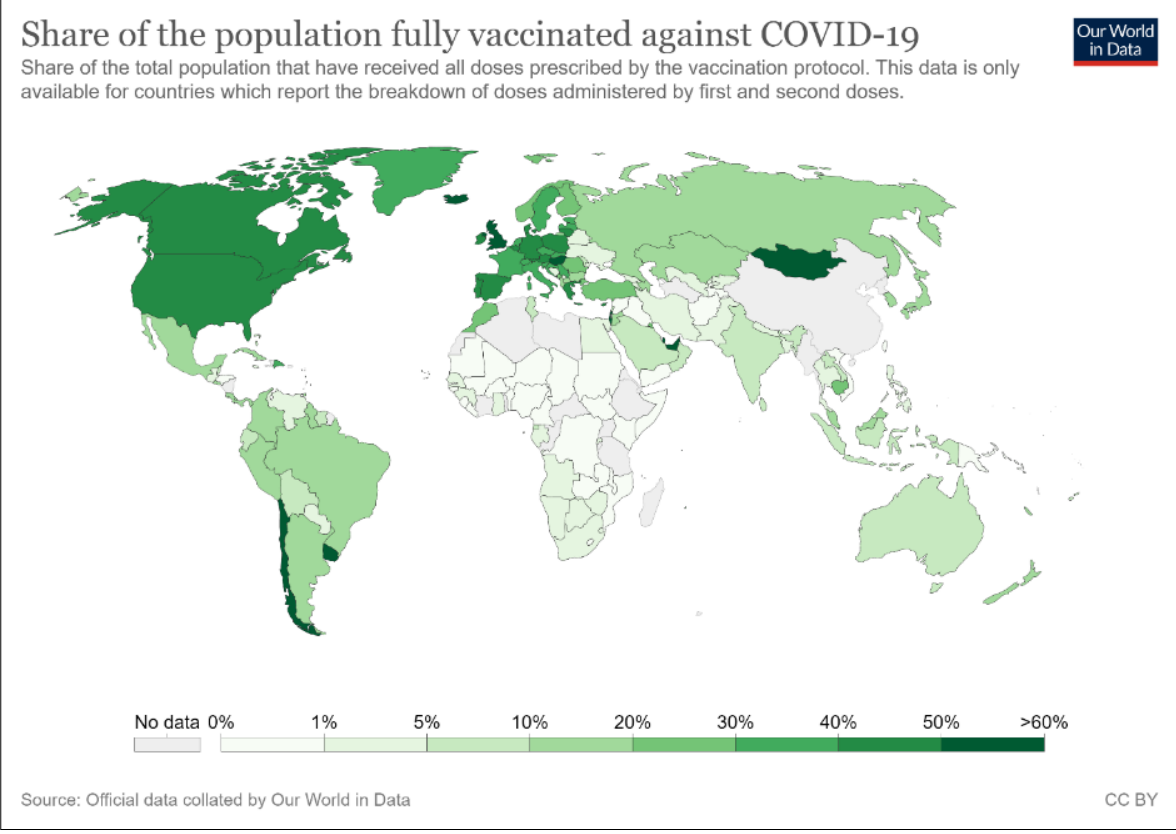

Figura 4. Planisfero che mostra con differenti colorazioni la percentuale di copertura vaccinale della popolazione nel mondo, aggiornata a luglio 2021. Fonte: (https://ourworldindata.org). 
del virus, proteggendo i soggetti più fragili ed evitando nuove ondate pandemiche.

Ma il vaccino non è tutto: le altre misure profilattiche non vanno accantonate, la popolazione deve essere sensibilizzata con strategie adatte al contesto e vanno sostenuti e portati avanti i servizi sanitari essenziali.

Vanno considerate problematiche estranee al contesto "occidentale": in contesti come quello africano non tutti gli Stati sono attrezzati per organizzare una campagna vaccinale di così ampio respiro per carenza di infrastrutture, personale e tecnologie che mantengano la catena del freddo. Non si può escludere che alcune fasce della popolazione vuoi per motivi culturali, religiosi o per la diffusione di false notizie rifiutino di vaccinarsi. Si incontrano realtà uniche e difficili come i campi profughi, popolazioni nomadi e aree colpite da conflitti interni e instabilità politica.

\section{Per concludere}

Un sistema che si basa unicamente sugli aiuti non sarà mai equo. È auspicabile quindi che questa diventi un'occasione per investire in aiuti a lungo termine ovvero nel potenziamento del sistema farmaceutico africano così come dei loro centri di ricerca, offrendo la nostra esperienza per porre le basi di un graduale svezzamento dal meccanismo delle donazioni e interrompendo la moderna dinamica economica di "global coloniality" [14].

\section{chiara.zavattero@auslromagna.it}

1. WHO Coronavirus (COVID-19) Dashboard. July 13, 2021. https://covid19.who.int. 2. Coronavirus (COVID-19). WHO, Regional Office for Africa. July 13, 2021. https:// www.afro.who.int/health-topics/coronavirus-covid-19.

3. Njenga MK, Dawa J, Nanyingi $M$, et al. Why is There Low Morbidity and Mortality of COVID-19 in Africa? Am J Trop Med Hyg. 2020 Aug;103(2):564-9.

4. Frost I, Craig J, Osena G, et al. Modelling COVID-19 transmission in Africa: countrywise projections of total and severe infections under different lockdown scenarios. BMJ Open. 2021 Mar 8;11(3):e044149.

5. Haider N, Osman AY, Gadzekpo A, et al. Lockdown measures in response to COVID-19 in nine sub-Saharan African countries. BMJ Global Health 2020; 5:e003319.

6. Wehbe S, Fahme SA, Rizk A, et al. COVID-19 in the Middle East and North Africa region: an urgent call for reliable, disaggregated and openly shared data. BMJ Global Health 2021;6:e05175.

7. Paremoer L, Nandi S, Serag H, et al. Covid-19 pandemic and the social determinants of health. BMJ. 2021 Jan 28;372:n129.
8. Bibi-Aisha Wadvalla. How Africa has tackled covid-19. The BMJ 16 July 2020.

9. Weekly bulletins on outbreaks and other emergencies. WHO, Regional Office for Africa. July 13, 2021. https://www.afro.who. int/health-topics/disease-outbreaks/outbreaks-and-other-emergencies-updates.

10. ArcGIS Dashboards. July 13, 2021. https://who.maps.arcgis.com/apps/dashboards/0c9b3a8b68d0437a8cf28581e9c063a9. 11. Shapira G, Ahmed T, Drouard SHP, et al. Disruptions in maternal and child health service utilization during COVID-19: analysis from eight sub-Saharan African countries. Health Policy Plan. 2021 Jun 19;czab064.

12. Michielsen K, Larrson EC, Kågesten A, et al. International Sexual Health And REproductive health (I-SHARE) survey during COVID-19: study protocol for online national surveys and global comparative analyses. Sex Transm Infect. 2021 Mar;97(2):88-92.

13. So AD, Woo J. Reserving coronavirus disease 2019 vaccines for global access: cross sectional analysis. BMJ. 2020 Dec 15;371:m4750. 14. Harman S, Erfani P, Goronga T, et al. Global vaccine equity demands reparative justice - not charity. BMJ Global Health 2021;6:e006504.

15. Un Panel di esperti esorta il G20 ad un "accordo globale" per prevenire i costi sociali ed economici delle future pandemie. July 9, 2021. https://www.g20.org.

16. Di cosa si parla al G7. Il Post. June 13, 2021. http://www.ilpost.it.

\section{COVID-19 nell'età pediatrica: non fermiamoci ai vaccini}

Qual è la conseguenza più grave della pandemia in corso sulla salute dei bambini? Un enorme studio di corte britannico ha raccolto i dati di 258.790 bambini tra marzo 2020 e febbraio 2021: 6.975 positivi al test SARS-CoV-2, durata media malattia 6 giorni con solo il 4,4\% degli ammalati con sintomi per 28 giorni o oltre (affaticamento, cefalea, anosmia); questi ultimi avevano un'età media di 14 anni e alla fine sono guariti completamente senza conseguenze [1].

Potremmo sentirci rasserenati da questi numeri se non fosse per una metanalisi che ha raccolto 29 studi pediatrici con oltre 80.000 partecipanti e che ha analizzato la prevalenza globale di depressione 0 ansia clinicamente rilevanti durante il corso della pandemia. Se le stime globali per depressione e ansia erano stimate essere rispettivamente dell' $8,5 \%$ e dell' $11,6 \%$ in epoca prepandemica, la metanalisi ha rivelato un tasso di depressione del $25 \%$ e del $21 \%$ per ansia in bambini e adolescenti: un aumento di prevalenza di oltre due volte. La prevalenza aumenta con l'età dei bambini e con l'aumento del numero dei mesi di pandemia, con l'ansia più frequente nelle femmine e nei Paesi europei. Anche se quasi la metà degli studi riguarda la Cina, la metanalisi è rappresentativa di ogni continente e di popolazioni di diverse estrazioni socioeconomiche; inoltre un'analisi di sensitività che ha escluso gli studi di bassa qualità (e con prevalenze più alte) ha confermato questi drammatici esiti [2]. In soldoni, quindi, alla fine del primo anno di pandemia 1 ragazzo/a su 4 a livello globale sta vivendo sintomi di depressione e 1 ragazzo/a su 5 soffre di ansia clinicamente rilevante.

In realtà questi sono dati che potrebbero non sorprendere chi lavora in ambulatorio, ossia in continuo contatto con i bambini e le famiglie: la sofferenza c'è ed è percepibile. Un problema per il medico è intercettare il bisogno di aiuto, spesso richiesto in modo improprio; in molti casi i genitori non informano il pediatra o il medico di medicina generale nonostante la gravità della situazione, più evidente soprattutto quando sono presenti disuguaglianze preesistenti, discriminazioni, ridotto accesso alla sanità e così via. Un altro grosso problema è l'accesso a cure e interventi di qualità ed efficaci: i maggiori bisogni di salute mentale emersi da questa pandemia al momento non trovano risposte nei nostri sistemi sanitari.

1. Molteni E, Sudre CH, Canas LS, et al. Illness duration and symptom profile in symptomatic UK school-aged children tested for SARS-CoV-2. Lancet Child Adolesc Health. 2021 Aug 3;S2352-4642(21)00198-X

2. Racine N, McArthur BA, Cooke JE, et al. Global Prevalence of Depressive and Anxiety Symptoms in Children and Adolescents During COVID-19: A Meta-analysis. JAMA Pediatr. 2021 Aug 9. 